\title{
Updated meta-analysis of comparison of mortality in enteral feeding (EN) vs. parenteral nutrition (PN) or other methods in gastrointestinal cancer patients
}

\author{
$\underline{\text { Al Bartolucci }^{a}}$, Sejong Bae ${ }^{\mathrm{b}}$ and Karan P. Singh ${ }^{\mathrm{b}}$ \\ ${ }^{a}$ Department of Biostatistics, School of Public Health, University of Alabama at \\ Birmingham, Birmingham, Alabama 35294, USA \\ ${ }^{b}$ Comprehensive Cancer Center, Division of Preventive Medicine, \\ School of Medicine, University of Alabama at \\ Birmingham, Birmingham, Alabama 35294, USA \\ Email:abartol@uab.edu
}

\begin{abstract}
Clinical trials and meta-analyses investigating the effect of enteral nutrition (EN), parenteral nutrition (PN) or other methods of nutritional support are at best confusing with multiple endpoints including major and minor complications as well as mortality. Most deal with a combination of diagnoses such as Crohn's disease, head trauma, liver disease, pancreatitis, various cancers, etc. and neglect to present separate results by diagnoses causing one to seek out the source documentation. There is no up to date comprehensive meta-analysis of EN vs. other methodologies in gastrointestinal (GI) cancer. The goal here is to compare EN vs. alternative methods in GI cancers only with respect to mortality endpoint.

A systematic literature (clinical trials and meta-analyses) search was performed for EN for the past 20 years using Pubmed, Medline, Cancerlit, Embase Cochrane collaborative plus other sources. Randomized clinical trials comparing EN to PN or other methods such as "nil by mouth", intravenous crystalloid solutions, and other oral supplementation were identified. The focus of the search was on articles from the cancer and clinical nutrition literature as well as abstracts and proceedings. This yielded about 14 such publications (1995-2012) with adequate data used in this analysis. Four studies had no deaths in both groups and are not presented here. The primary endpoint for our meta-analysis was incidence of mortality given the raw death incidence and sample size. Many publications provide information on the reduced risk of death of one intervention vs. the other which could be put into a meta-regression with the accumulated data from other publications.
\end{abstract}

We used a Bayesian random effects model to account for heterogeneity across the studies. However, considering the restricted diagnosis of the subject pool and the similarity of treatment approaches, heterogeneity was not anticipated to be significant. The priors used were non informative and conjugate on the prior mean and variance of the odds ratio (OR) yielding a distribution for both the odds ratio and the predictive odds ratio. We also investigated plots of the distributions of these statistics.

Of the data analyzed, the odds ratio (OR) of mortality in GI cancer of EN vs. other methods is about 0.819 with 95\% confidence interval CI, $(0.571,1.174)$ and the predictive OR is about 0.893 , with 95\% CI (0.546, 1.230). The posterior distributional plots of the OR and predictive OR yield interesting patterns over the studies. The heterogeneity as anticipated was non-significant.

Assuming a wide distributional effect of EN over other methodologies there appears to be a consistent trend that, although EN is preferred because of other reduced morbidities in many publications, the effect on overall mortality of the reduced risk evidenced by this analysis is not statistically convincing across studies. However, using a Bayesian analysis, the posterior probability of the OR being less than one (in favor of EN) ranges from 0.811 to 0.99 and the predicted OR being less than one ranges from 0.544 to 0.888 for realistic prior ranges of the OR over time. On a final note, although EN is superior to other methods with respect to mortality, the effect of this superiority has appeared to decrease over time as seen in the last plot of this manuscript.

Keywords: Enteral Feeding, Parenteral Nutrition, Gastrointestinal Cancer, Clinical trials, Meta-Analyses, Bayesian Random Effects, Odds Ratio. 
Bartolucci et al., Updated meta-analysis of comparison of mortality in enteral feeding (EN) vs. parenteral nutrition (PN) or other methods in gastrointestinal cancer patients

\section{INTRODUCTION}

There are a number of references comparing the effect of various feeding methods on the well being of subjects with many types of diseases. Braunschweig et al (2001) present an excellent overview of the technique known as enteral feeding (EN) vs. other methodologies including parenteral nutrition (PN). They note that parenteral nutrition is an invasive therapy that provides nutrition support for individuals who do not have adequate gastrointestinal functions; however, it does have inherent risks such as pneumothorax, vomiting, diarrhea constipation and several catheter complications or injuries. Enteral nutrition, specifically tube feeding, is the preferred method of feeding because it is less expensive, has fewer complications, and has better outcomes in terms or morbidity than parenteral nutrition. A review of the prospective randomized clinical trials (PRCTs) that compared tube feeding with parenteral nutrition cast doubt on some of these generally accepted benefits. They conducted a rather extensive meta-analysis examining the benefits of EN over PN. They note that previous reviews, although comprehensive, did not systematically compile results or evaluate the quality of the studies' methods, and they also included many studies conducted in populations that would not be considered candidates for parenteral nutrition by today's standards, although they were considered critically ill subjects. Heyland et al. (1998) conducted a meta-analysis of PRCTs that evaluated the outcomes of parenteral nutrition compared with those of standard care (conventional oral diets with intravenous dextrose) in surgical or critically ill patients. They found that parenteral nutrition did not influence a mortality rate which is a focus of this paper However, a trend toward fewer complications; particularly in populations that had protein-energy malnutrition was reported. Their analysis included studies that provided parenteral nutrition in amounts less than the estimated energy and protein needs of the patients and trials conducted in populations with functional gastrointestinal tracts. Both of these factors, according to Braunschweig et al. (2001), could have reduced the influence of parenteral nutrition on outcomes. Also, they did not include investigations that compared parenteral nutrition with tube feeding.

Most studies comparing EN to other methods deal with a combination of diagnoses such as Crohn's disease, head trauma, liver disease, pancreatitis, various cancers, etc. and neglect to present separate results by diagnoses causing one to seek out the source documentation. There is no up to date comprehensive metaanalysis of EN vs. other methodologies in GI cancer. The purpose of this study was to review systematically and aggregate statistically the PRCTs that were conducted in populations appropriate for random assignment to parenteral nutrition or other methods to the more accepted EN (tube feeding) and limited nutritional intervention (standard care) on mortality. Thus the overall question as to whether or not EN is advantageous over other feeding methodologies.

One must keep in mind that the GI physiology is important no matter what the feeding intervention. Parenteral nutrition is designed to provide nutrition to patients who cannot be nourished adequately by enteral nutrition

for a critical period of time. The time period can vary from several days to weeks. Because of the inherent risk and higher cost of parenteral nutrition, it is generally not used as a substitute for enteral nutrition if either standard care or tube feeding is feasible. Also, because of physiologic changes that are caused by gastrointestinal tract dysfunction, outcomes observed in populations with functional gastrointestinal tracts might be different from those observed in populations without adequate gastrointestinal function. The criteria and complications met by the GI cancer populations included in the meta-analysis are varied. They include, but are not limited to, mal-absorptive syndromes with severe food, electrolyte, and fluid losses, severe shortbowel syndrome not adequately managed by oral or enteral nutrition. The syndrome can be induced by infection, inflammatory, and immunologic disorders, drugs, or radiation, high-output gastrointestinal fistulas that enteral intubation cannot bypass or severe renal tubular defects with large fluid and ion losses. There are motility disorders such as persistent ileus (postoperative or disease related), severe intestinal pseudoobstruction, severe persistent vomiting caused by medication, brain tumor, or other disorder (eg, hyperemesis gravidarum). Other criteria included mechanical intestinal obstruction not immediately remedied by surgery, perioperative state with severe under nutrition and critically ill patients, especially those with hypermetabolism, who are not appropriate for enteral nutrition because it was contraindicated or failed.

Tube feeding or EN was defined as either surgical or nonsurgical placement of a small flexible tube into the gastrointestinal tract to provide required nutrients. Standard care was defined as the gradual reintroduction of an oral diet as tolerated after its interruption was caused by a disease or a surgical procedure that resulted in several days of inadequate nutrient intake and the use of intravenous dextrose or fluids for hydration. It could include "nil by mouth”, intravenous crystalloid solutions, and other oral supplementation. 
Bartolucci et al., Updated meta-analysis of comparison of mortality in enteral feeding (EN) vs. parenteral nutrition (PN) or other methods in gastrointestinal cancer patients

\section{METHODS}

The eighteen trials from which we gathered the data for our analyses have been published, outlined, and examined individually for their mortality rates with either EN or non EN intervention. They are too numerous to describe individually, but are referenced at the end of this manuscript. Four of the studies had no deaths and are not include in the meta-analysis. For the purposes of a meta-analysis comparing the mortality of EN vs. non EN intervention, the task is to apply a binomial sampling model to the data. From the model we derive the logit of the response (failure,i.e. death) and compute the posterior odds ratio of response for each study and then derive the overall odds ratio for the 14 trials using meta-analysis. Once this is done then we derive the posterior predicted odds ratio for the group. The authors have utilized the MCMC procedure for deriving the posterior parameters of the model which include the posterior odds and predicted posterior odds. We let $\mathrm{rC}_{\mathrm{i}}$ and $\mathrm{ra}_{\mathrm{i}}$ denote the number of failures in the control group (non EN) and active group (EN), respectively in each of the 14 studies, $\mathrm{i}=1, \ldots, 14$. Likewise we let $\mathrm{nc}_{\mathrm{i}}$ and $\mathrm{na}_{\mathrm{i}}$ be the total number of subjects in the control and active groups respectively, $i=1, \ldots, 14$. We further define

$$
\begin{aligned}
& \mathrm{rc}_{\mathrm{i}} \sim \operatorname{binomial}\left(\mathrm{pc}_{\mathrm{i}}, \mathrm{nc}_{\mathrm{i}}\right), \\
& \mathrm{ra}_{\mathrm{i}} \sim \operatorname{binomial}\left(\mathrm{pa}_{\mathrm{i}}, \mathrm{na}_{\mathrm{i}}\right)
\end{aligned}
$$

and,

where

$$
\begin{aligned}
& \operatorname{logit}\left(\mathrm{pc}_{\mathrm{i}}\right)=\mu_{\mathrm{i}} \\
& \operatorname{logit}\left(\mathrm{pa}_{\mathrm{i}}\right)=\mu_{\mathrm{I}}+\operatorname{delta}_{\mathrm{i}}
\end{aligned}
$$

$$
\mu_{\mathrm{i}} \sim \operatorname{normal}((0.0,1.0 \mathrm{E}-5)
$$

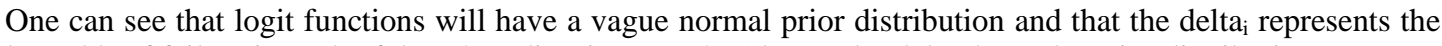
$\log$ odds of failure in each of the 14 studies, $i=1, \ldots, 14$. Also we let delta ${ }_{i}$ have the prior distribution,

$$
\text { delta }_{\mathrm{i}} \sim \mathrm{t}(\mathrm{d}, \mathrm{tau}, 4)
$$

which is a t distribution which hyper prior mean $\mathrm{d}$, inverse variance, tau, variance sigma=1/tau, and degrees of freedom, 4. One can see that this is a rather flat distribution with only 4 degrees of freedom. The mean, d, is actually the overall mean of the 14 studies combined and $d$ and tau have the hyper prior vague distributions,

$$
\begin{aligned}
& \mathrm{d} \sim \operatorname{normal}(0.0,1.0 \mathrm{E}-6) \\
& \text { tau } \sim \operatorname{gamma}(0.001,0.001) .
\end{aligned}
$$

The odds ratio or odds of failure for the treated group versus the control group for each of the 14 studies is,

$$
\text { odds }_{\mathrm{i}}=\exp \left(\text { delta }_{\mathrm{i}}\right)
$$

with overall odds for the 14 studies combined having the value,

$$
\text { odd_R }=\exp (\mathrm{d}) \text {. }
$$

We define one more parameter, or the predicted value of the odds, which is simply,

$$
\text { Odd_Pred =exp(delta.new). }
$$

The key advantage of using a Bayesian approach for the EN vs. non EN trials is the ability of the Bayesian inferential approach to incorporate background or empirical information thought relevant to the clinical question being addressed ( Bartolucci et al. 2008).

\section{RESULTS}

Table 1 represents the Forest Plot of the main results by authors last initial and year of publication. Some studies are imbedded in the references cited below. Of the data analyzed, the odds ratio (OR) of mortality in 
Bartolucci et al., Updated meta-analysis of comparison of mortality in enteral feeding (EN) vs. parenteral nutrition (PN) or other methods in gastrointestinal cancer patients

GI cancer of EN vs. other methods is about 0.819 with $95 \%$ confidence interval CI, $(0.571,1.174)$ and the predictive OR was computed to be about 0.893 , with $95 \%$ CI $(0.546,1.230)$. The posterior distributional plots of the OR and predictive OR yield interesting patterns over the studies. The heterogeneity was nonsignificant.

Table 1 Forest Plot of the 14 Studies in the Meta-Analysis

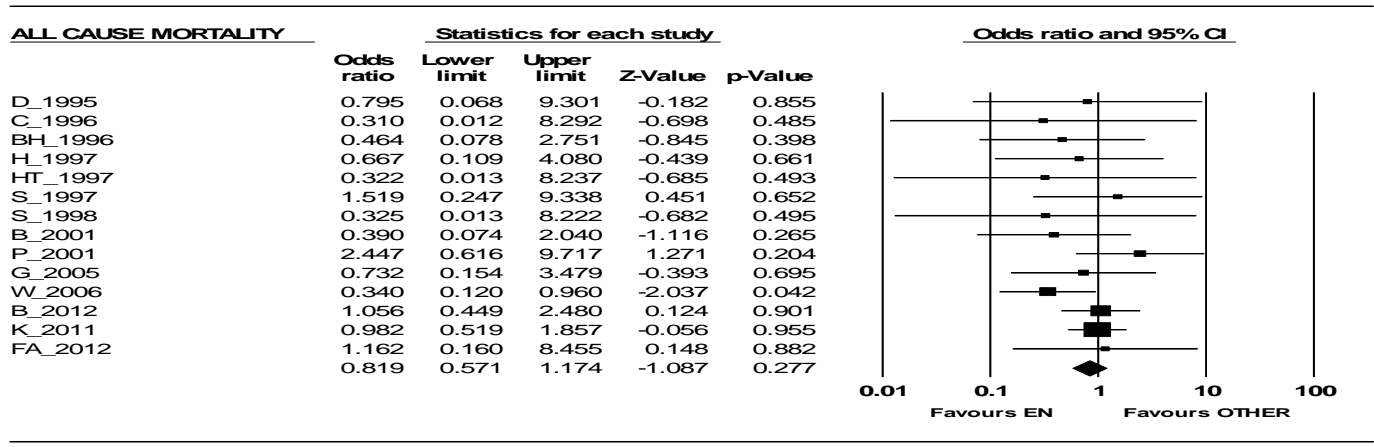

Meta Analysis

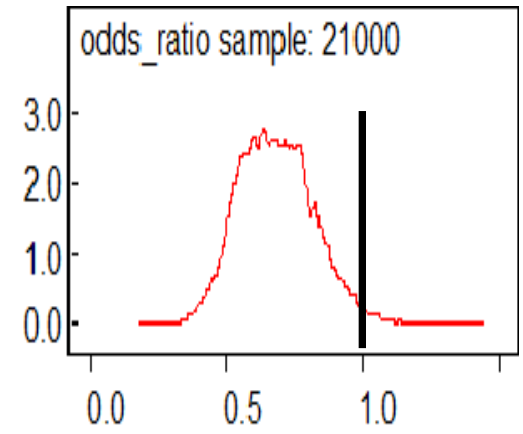

Figure 1. Posterior Odds Ratio
We next examined the posterior probability plots of the odds ratio and predicted odds as is seen in Figures 1 and 2. One can note at the horizontal axis that the bulk of the posterior density is to the left of the value 1 . The value 1indicated by the vertical line represents equal odds of failure. The EN group is in the numerator of the odds ratio. Thus one can see that the posterior probability of the odds ratio and the posterior probability of predicted odds ratio are much less than one. When one examines Figure 2, the results are much the same. Tables 2 and 3 give the results of the values of the odds ratio and predicted odds ratio for a robust range of prior inputs. The odds ratio varies from 0.574 to 0.841 indicating a much lower odds of failure on the EN vs. non EN interventions. The posterior probability that the distribution is less than one or less than equal odds of failure for the EN group is quite large ranging from 0.811 to 0.999 . Similarly for Table 4 the posterior predicted odds ranges from 0.597 to 0.893 with a range of probability from 0.544 to 0.888 that the odds is less than one.

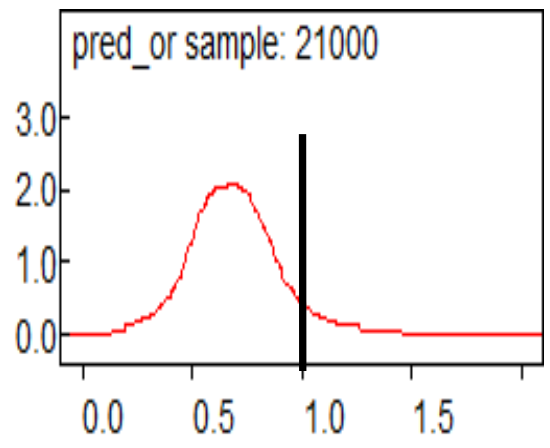

Figure 2. Posterior Predicted Odds Ratio
Table 2. Tabulated Posterior Odds Ratios

\begin{tabular}{|c|c|c|}
\hline OR & Variance & $\begin{array}{c}\text { Probability } \\
\text { That OR Less } \\
\text { Than 1 }\end{array}$ \\
\hline 0.841 & 0.180 & 0.811 \\
\hline 0.746 & 0.152 & 0.953 \\
\hline 0.695 & 0.138 & 0.987 \\
\hline 0.629 & 0.132 & 0.997 \\
\hline 0.602 & 0.122 & 0.999 \\
\hline 0.574 & 0.123 & 0.999 \\
\hline
\end{tabular}


Bartolucci et al., Updated meta-analysis of comparison of mortality in enteral feeding (EN) vs. parenteral nutrition (PN) or other methods in gastrointestinal cancer patients

Table 3. Tabulated Posterior Predicted Odds Ratios (OR)

\begin{tabular}{|l|l|l|}
\hline OR & Variance & $\begin{array}{l}\text { Probability That } \\
\text { Predicted Odds } \\
\text { Less Than 1 }\end{array}$ \\
\hline 0.893 & 0.966 & 0.544 \\
\hline 0.796 & 0.603 & 0.632 \\
\hline 0.724 & 0.418 & 0.745 \\
\hline 0.674 & 0.379 & 0.805 \\
\hline 0.623 & 0.356 & 0.855 \\
\hline 0.597 & 0.332 & 0.888 \\
\hline
\end{tabular}

Upon examining the Forest plot of Table 1 a bit closer one notes that the positive effect of EN (i.e. OR<1) actually decreases from 1995 to 2012. Thus although examining all the studies there appears to be a superiority of EN over other feeding methods in general, the trend is not consistent. Taking a moving average of the OR vs. log year from 1995 to 2012 the plot in Figure 3 indicates that the likelihood of the OR trending greater than one over time decreases. This was an unexpected finding after examining the results. The overall trend was significant, $\mathrm{p}=0.0098$.



Figure 3. Moving Average of OR from 1995-2012.

\section{DISCUSSION AND CONCLUSIONS}

One can always question the quality of the studies in a meta-analysis. Because differences in study populations and design might cause variations in results, some sources for heterogeneity were defined a priori such as study-quality score, year of study publication, nutritional status of patients, and percentage of patients with cancer. The method used in each study was evaluated for the quality of these characteristics: appropriate randomization, comparability of groups at baseline, endpoints (blinded to staff or not), well described treatment protocols, well-defined outcomes, and analysis by intent to treat. The heterogeneity statistic was non -significant which indicates that the investigators did reasonably well putting together these studies for such a very narrow focus, i.e. mortality in the feeding comparison of EN vs. other methodologies. As pointed out by Braunschweig et al (2001), it is well known that the skill with which specialized nutrition support is provided has improved since it was first used in patient care. The decreasing trend for superiority of the EN as noted in Figure 3 above may be an indication that the skill factor is important over time.

Unlike previous studies a goal of this meta-analysis was not to provide an interim guide for clinical decisionmaking until the results of large trials conducted in populations with gastrointestinal dysfunction are available. A comprehensive search of the literature for studies with a mortality endpoint was done with the use of clinically relevant criteria for both study selection and assessed outcomes. In a broad spectrum of patients with compromised gastrointestinal function with tube feeding, others have found fewer infections in those who were tube-fed than in those who received parenteral nutrition. These findings were similar, although not as strong, in a comparison of standard care with parenteral nutrition in normally nourished patients. It is important to note that Braunschweig at al (2001) also found a higher risk of nutrition support complications with tube feeding than with parenteral nutrition. Accordingly, these results suggest that waiting 7-10 days to initiate any form of aggressive nutrition intervention may be prudent for normally nourished populations with compromised gastrointestinal function. Further longer term studies that compare outcomes of tube feeding with those of standard care in both normally nourished and malnourished populations are needed. Finally, one must pursue a cost endpoint in studies comparing either tube feeding or parenteral nutrition with those of standard care to guide clinicians, hospital administrators, and third-party payers in their decision-making. 
Bartolucci et al., Updated meta-analysis of comparison of mortality in enteral feeding (EN) vs. parenteral nutrition (PN) or other methods in gastrointestinal cancer patients

Our purpose was to determine in aggregate setting which method (EN vs. other) seems to fare better with overall mortality as an endpoint. Many of these subjects are fairly well advanced in their diagnosis and one may question the relevancy of pursuing this goal. However, survival continues to be a primary endpoint in many clinical studies and it is thus important to continue to examine the statistical tools available to reach a consensus on the most beneficial approach.

The authors have utilized the MCMC procedure for deriving the posterior parameters of the model which include the posterior odds and predicted posterior odds. It was important based on previous survival studies to attempt a reasonable posterior predicted odds ratio for the groups being compared and hopefully confirm the comfort level with which investigators have felt in using enteral nutrition in the appropriate patient populations. The Bayesian methodology is a reasonable approach to help update and summarize the knowledge to date in this clinical application..

\section{ACKNOWLEDGMENTS}

Research reported in this publication was partially supported by the National Cancer Institute of the National Institutes of Health to the University of Alabama at Birmingham Comprehensive Cancer Center under award number P30 CA013148. The content is solely the responsibility of the authors and does not necessarily represent the official views of the National Institutes of Health.

\section{REFERENCES}

Baldwin, C., Spiro, A., Ahern,R, Emery, P.W. (2012). Oral nutritional interventions in malnourished patients with cancer: A systematic review and meta-analysis. Journal National Cancer Institute,104, 1-15.

Bartolucci, AA. Bae, S.J. and Singh, K.P. (2008). Establishing a Bayesian predictive survival model adjusting for random effects. Mathematics and Computers in Simulation, 78, 328-334.

Beier-Holgersen, R.,Boesby,S. (1996). Influence of postoperative enteral nutrition on postsurgical infections. British Medical Journal/Gut, 39, 833-835.

Bozzetti, F.,Braga, M., Gianotti, L, Cavazzi,, C., Mariani, L. (2001). Postoperative enteral versus parenteral nutrition in malnourished patients with gastrointestinal cancer; a randomized multicenter trial. The Lancet. 358. 1487-1492.

Braga, M., Vignali, A., Gianotti, L., Cestari,, A., Profili, M., DiCarlo, V. (1995). Benefits of early postoperative enteral feeding in cancer patients. Infusion Therapy: Transfusions Med, 22, 280-284.

Braga, M., Gianotti, L., Vignali, A., Cestari, A., Bisagni, P., M., DiCarlo, V. (1998). Artificial nutrition after major abdominal surgery: impact of route of administration and composition of the diet, Critical Care Medicine, 26, 24-30.

Braunschweig, C., Levy, P., Sheean, P.M., Wang, X. (2001). Enteral compared with parenteral nutrition: a meta-analysis, American Journal of Clinical Nutrition, 74, 534-542.

Carr, CS., Ling KD, Boulos P, Singer, M. (1996). Randomized trial of safety and efficacy of immediate postoperative enteral feeding in patients undergoing gastrointestinal resection., British Medical Journal, 312. 869-871.

Daly, J.M., Weintraub, F., Shou, J., Rosato, EF., Lucia, M. (1995). Enteral nutrition during multimodality therapy in upper gastrointestinal cancer patients, Annals of Surgery, 221(4), 327-338.

Fujita, T., Daiko H. Nishimura M. (2012). Early enteral nutrition reduces the rate of life-threatening complications after thoracic esophagectomy in patients with esophageal cancer. European Surgical Research, 48(2), 79-84..

Fujitani,K., Tsujinaka, T., Fujita, J., Miyashiro, I., Imamura, H., Kimura, Y., Kobayashi, K., Kurokawa, Y.,. Shimokawa, T., Furukawa, H. (2012). Prospective randomized trial of preoperative enteral immunonutrition followed by elective total gastrectomy for gastric cancer, British Surgical Journal, 99(5), 621-629.

Gabor, S., Renner, H., Matzi, V., Ratzenhofer, B., Lindenmann, J., Sankin, O., Pinter, H. Maier, a., Smolle, J., Smolle-Juttner, F.M. (2005), Early eneral feeding compared with parenteral nutrition after oesophageal or oesophagogastric resection and reconstruction, British Journal of Nutrition, 93, 509-513.

Hartsell, PA, Frazee, RC., Harrision, JB.,Smith, RW. (1997), Early postoperative feeding after colorectal surgery, Arch. Surgery,132, 518-521.

Heslin, MJ., Larkeny, I., Leung, D.,Brooks, AD.,Hochwald, SN., Pisters, PW. (1997). A prospective randomized trial of early enteral feeding after resection of upper gastrointestinal malignancy, Annals of Surgery, 226(4), 567-580.

Heyland, DK., MacDonald, S., Keefe, L., Drover, JW. (1998). Total parenteral nutrition in the critically ill patient, JAMA, 280, 2013-2019. 
Bartolucci et al., Updated meta-analysis of comparison of mortality in enteral feeding (EN) vs. parenteral nutrition (PN) or other methods in gastrointestinal cancer patients

Klek, S., Sierzega, ., Szybinski, P., Szczepanek, K., Scislo, L., Waleewska, E., Kulig, J. (2011). Perioperative nutrition in malnourished surgical cancer patients - a prospective, randomized, controlled clinical trial, Clinical Nutrition,30, 708-713.

Pacelli, F., Bossola, M., Papa, V., Malerba, M., Modesti, C., Sgadari, A., Bellantone, R., Doglietto, G., (2001). Enteral vs. parenteral nutrition after major abdominal surgery. Archives Surgery, 136. 933-936.

Senkal, M., Mumme, A., Eickoff, U. (1997). Early postoperative enteral immunonutrition: clinical outcome and cost comparison analysis in surgical patients. Critical Care Medicine, 25, 1489-1496.

Stewart BT., Woods RJ., Collopy BT., Fink RJ., Mackay JR., Keck JO. (1998). Early feeding after elective open colorectal resections: a prospective randomized trial, Aust. NZJ Surgery, 68, 125-128.

Wu, GH., Liu, ZH., Wu, ZG. (2006). Perioperative artificial nutrition in malnourished gastrointestinal cancer patients, World Journal of Gastroenterology, 12(15), 24441-2444. 